\title{
Investigating bloodstain dynamics at impact on the technical rear of fabric
}

\author{
Dicken, L. ${ }^{1}$, Knock, C. ${ }^{1}$, Carr, D. J. ${ }^{2}$, Beckett, $\mathrm{S}^{3}$
}

\section{Authors' addresses}

${ }^{1}$ Centre for Defence Engineering, Cranfield University at the Defence Academy of the United Kingdom, Shrivenham, SN6 8LA, United Kingdom.

${ }^{2}$ Formerly at Centre for Defence Engineering, Cranfield University at the Defence Academy of the United Kingdom, Shrivenham, SN6 8LA, United Kingdom. Now at Defence and Security Accelerator, Porton Down, Salisbury, Wiltshire, SN4 OPQ.

${ }^{3}$ Cranfield Forensic Institute, Cranfield University at the Defence Academy of the United Kingdom, Shrivenham, SN6 8LA, United Kingdom.

\section{Communicating Author}

Dr. C. Knock, Centre for Defence Engineering, Cranfield University at the Defence Academy of the United Kingdom, Shrivenham, SN6 8LA, United Kingdom.

Telephone: +44 (0)1793 785335

Email: c.knock@cranfield.ac.uk

\begin{abstract}
Using high speed video, the impact of blood drops falling at three velocities (1.9, 4.2 and $\left.5.8 \mathrm{~ms}^{-1}\right)$ were filmed from both the technical face and for the first time the technical rear of three different mass per unit areas $\left(85.1,163.5\right.$ and $\left.224.6 \mathrm{~g} / \mathrm{m}^{2}\right)$ of $100 \%$ cotton calico. It was seen that there were two stages in the creation of a bloodstain on fabric; the impact dynamics, followed by wicking along the intra-yarn spaces. In the first stage, once the blood impacted the fabric, blood was visible on the technical rear of the fabrics with the medium and lightest mass per unit area within as little as 0.067 ms after impact. No blood was visible on the technical rear of the fabric with the heaviest mass per unit area following impact or the medium mass per unit area from $1.7 \mathrm{~ms}^{-1}$ impacts. On the technical face of the fabric, the blood drop spread laterally and then receded for $8 \mathrm{~ms}$ following impact. The dynamics on the technical face were not affected by what was occurring on the technical rear of the fabric. The bloodstain on the technical rear initially only increased until $0.8 \mathrm{~ms}$ following impact. The increase in technical rear bloodstain area was caused by continued movement of the blood through to the rear of the fabric as the blood drop spread on the technical face. Once the impact dynamics were concluded within $8 \mathrm{~ms}$ of impact, there was no further change in the bloodstain for the remaining $67 \mathrm{~ms}$ of high speed video. Following this the blood wicked into and along the yarns, resulting in a dry technical rear bloodstain on all fabrics at all velocities.
\end{abstract}

\section{Keywords}

- Bloodstain analysis

- High speed video 
- Impact dynamics

- Plain woven fabric

- Technical rear

\section{Highlights}

- Blood penetrated to the technical rear of the fabric prior to surface spreading.

- $\quad$ The penetrated blood spread for no more than $0.6 \mathrm{~ms}$ after impact.

- Penetration of blood to the technical rear was fabric and velocity dependent.

- Wicking of the blood led to a dry technical rear bloodstain for all fabrics.

\section{Introduction}

In recent years research in blood pattern analysis (BPA) has increased rapidly [1-6]. An area which has been investigated within BPA is the impact dynamics of a blood drop on fabric. The appearance of a bloodstain following vertical impact onto a fabric surface can vary widely depending on the impact velocity and the fabric itself [1,3]. When porcine blood was allowed to fall vertically $200 \mathrm{~mm}$ onto $100 \%$ cotton plain woven and jersey knit fabrics, a number of stages were seen to occur following impact [4]. The drop spread on the surface of the fabric, followed by a crown and ligaments and satellite stains ${ }^{1}$ forming. The drop then retracted before stabilising. The drop area remained nearly constant for $0.5 \mathrm{~s}$ before increasing again owing to lateral wicking. The amount of wicking which would occur following impact would depend on the structure of the fabric and the yarns and the type of fibres, but could result in the bloodstain remaining small and circular, or increasing in size, becoming more irregular and possibly absorbing the spines and satellite stains $[1,3]$. What was not described in this previous work was what occurred on the technical rear of the fabric, and whether this affected the dynamics on the technical face.

The two most utilised mounting methods within BPA research are either fixing the fabric to a hard surface (such as foam core board) [1,2,4] or mounting the fabric on an embroidery hoop [3,4,7]. Research has shown that the surface on which the fabric is mounted for BPA research affects the impact dynamics of the blood drop, and therefore the resultant dry bloodstain. Passive bloodstains were created by dropping blood from between 100 and $110 \mathrm{~cm}$ onto three different fabrics $(100 \%$ cotton plain woven bed sheeting, technical face and technical rear of cotton single jersey knit t-shirt fabric and $100 \%$ cotton denim) [5]. No impact velocities were provided. For a 'hard' surface, the fabrics were placed directly onto a piece of blotting paper on a concrete floor. The 'hard' surface resulted in the drop initially spreading on the surface of the fabric before retracting and spines and satellite stains forming. The blood then 'wicked' into the textile, merging with many of the surrounding spines and satellite stains [5]. It has been suggested [3] that the use of a hard backing can create capillary bridges of blood between the fabric and the hard surface and affect the wicking pattern.

For a 'semi-hard' surface the woven and knit fabrics were placed on top of denim on the concrete floor. This softened the impact, resulting in fewer satellite stains and spines than for the hard surface.

\footnotetext{
${ }^{1}$ 'A smaller bloodstain that originated during the formation of the parent stain as a result of blood impacting a surface'. Scientific Working Group on Bloodstain Pattern Analysis, Recommended Terminology, (2015). https://hemospat.com/bloodstain-pattern-analysis-terminology/ (accessed May $8^{\text {th }}$ 2019).
} 
For a taut mounting, the woven and knit fabrics were stretched tautly in an embroidery hoop. The tautness was not measured, even though different levels of stretching could lead to different bloodstains owing to both the tautness of the fabric and the increase in the inter-yarn spaces. The fabrics were draped over the embroidery hoop for a 'loose' mounting. For a 'taut' mounting, some of the impacting blood was seen to bounce off the fabric, altering the bloodstain. This has also been reported to occur when fabrics were mounted on foam core board [8]. For the 'loose' mounting the fabric deformed and absorbed much of the kinetic energy, resulting in very few spines and satellite stains. In this previous work [5] bloodstains which resulted on the opposite side of the fabric to that impacted were not described, and only mentioned in terms of blood passing through the fabric resulting in bloodstains on the backing material.

The impact dynamics on the technical face of the fabric are well understood $[4,8]$. However, how the impact dynamics are affected by what is occurring on the technical rear of the fabric is not as well understood. The aim of the current work is to determine what dynamics occur on the technical rear of the fabric as the blood impacts the technical face. Two high speed cameras were used in this work to simultaneously video the technical face and technical rear of the fabrics.

\section{Materials and methods}

\section{Materials}

Three calico fabrics ( $100 \%$ cotton, plain woven ${ }^{2}$ ) were chosen for the experiments $\left(85.1 \mathrm{~g} / \mathrm{m}^{2}\right.$ (light), $163.5 \mathrm{~g} / \mathrm{m}^{2}$ (medium) and $224.6 \mathrm{~g} / \mathrm{m}^{2}$ (heavy)). The fabric specimens were prepared as per [8]. Briefly, the fabrics were made dimensionally stable by laundering six times on a cotton cycle at $40^{\circ} \mathrm{C}$ [9] before line drying and pressing to remove creases. The fabrics were cut into $100 \times 100 \mathrm{~mm}$ specimens [10] which were then conditioned for 24 hours at $20 \pm 2{ }^{\circ} \mathrm{C}$ and $65 \pm 4 \%$ relative humidity [11].

Fabric thickness [12], mass per unit area [13] and sett [14] were measured. Yarn linear density was estimated by the removal of ten $1 \mathrm{~m}$ long yarns from the fabric and weighing them using an Oxford A2204 balance accurate to four decimal places (table 1). SEM images of the fabrics at 50x magnification and the warp and weft yarns at $75 x$ magnification are available in the electronic supplementary material.

\begin{tabular}{|c|c|c|c|c|c|c|}
\hline & \multicolumn{2}{|c|}{ Light calico } & \multicolumn{2}{|c|}{ Medium calico } & \multicolumn{2}{|c|}{ Heavy calico } \\
\hline Thickness (mm) & \multicolumn{2}{|c|}{$0.38 \pm 0.03$} & \multicolumn{2}{|c|}{$0.46 \pm 0.02$} & \multicolumn{2}{|c|}{$0.56 \pm 0.03$} \\
\hline $\begin{array}{l}\text { Mass per unit area } \\
\left(\mathrm{g} / \mathrm{m}^{2}\right)\end{array}$ & \multicolumn{2}{|c|}{$85.1 \pm 1.54$} & \multicolumn{2}{|c|}{$163.5 \pm 2.26$} & \multicolumn{2}{|c|}{$224.61 \pm 1.56$} \\
\hline Sett (yarns per $10 \mathrm{~mm}$ ) & \multicolumn{2}{|l|}{$27 \times 23$} & \multicolumn{2}{|c|}{$25 \times 26$} & \multicolumn{2}{|c|}{$26 \times 26$} \\
\hline Yarn type $^{3}$ & \multicolumn{2}{|c|}{ Ring spun } & \multicolumn{2}{|c|}{ Ring spun } & \multicolumn{2}{|c|}{ Ring spun } \\
\hline & Warp: & Weft: & Warp: & Weft: & Warp: & Weft: \\
\hline Twist (turns per $\mathrm{m})^{1}$ & 650 & 650 & 756 & 756 & 650 & 624 \\
\hline Linear density (tex) & 14 & 18 & 33 & 31 & 43 & 47 \\
\hline
\end{tabular}

\footnotetext{
2 'The simplest of all weave interlacings in which the odd warp threads operate over one and under one weft thread throughout the fabric with the even weft threads reversing this order to under one, over one, throughout'. Textile Terms and Definitions (2002) 11th edition (revised) edited by Denton, M.J. and Daniels, P.N.

${ }^{3}$ Yarn type and twist were provided by the fabric company; Whaleys Bradford Ltd, Harris Court, Great Horton, Bradford, West Yorkshire, BD7 4EQ http://www.whaleys-bradford.Itd.uk
} 
Table 1 mean fabric properties and standard deviations where available $(n=5)$.

Defibrinated horse blood was sourced from Southern Group Laboratory ${ }^{4}$ to create the bloodstains. Horse blood properties have previously been found to be equivalent to human blood, [15] and horse blood is frequently used in lieu of human blood owing to health and safety considerations [16]. The blood was stored below $4^{\circ} \mathrm{C}$, and was used within one week of delivery.

\section{Methods}

A diagram of the set-up of the cameras and fabric specimen is given in figure 1. A phantom V12 highspeed video was mounted vertically beneath a stand with a clear polycarbonate surface. A second high-speed video, a Phantom V7, was mounted above the surface at an angle of $45^{\circ} 21^{\prime}$. This allowed simultaneous filming of the face and rear of the drop as it impacted the surface. The cameras were connected to the same trigger so both would trigger at the same time.

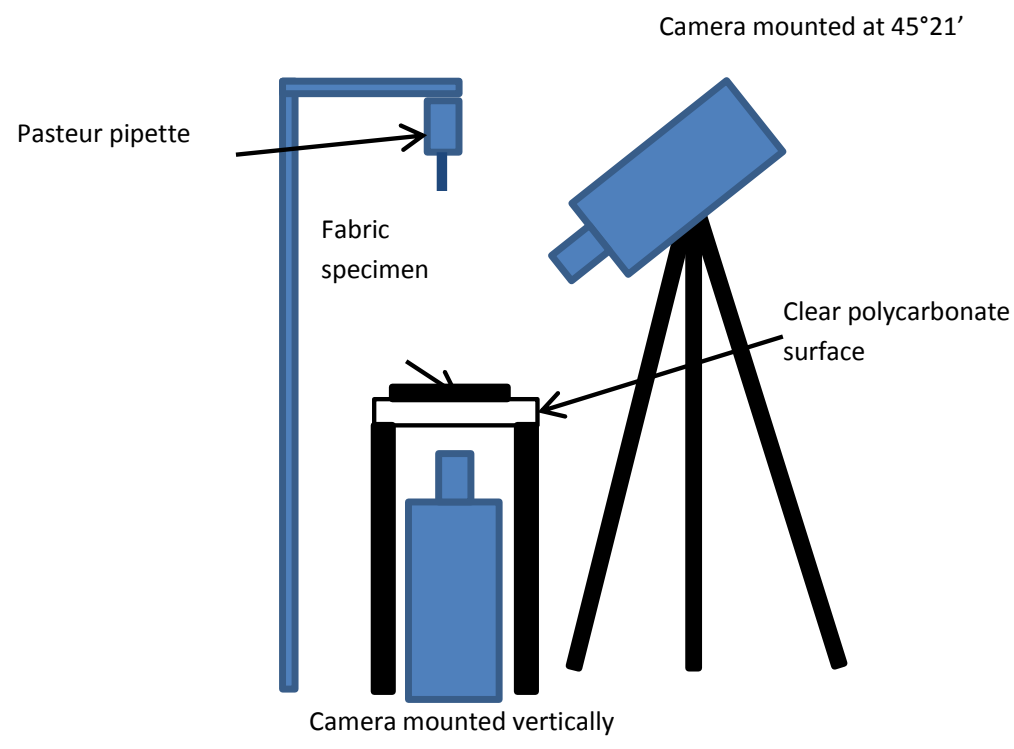

Figure 1 camera set-up

The camera settings are given in table 2 . The frame rate for the technical rear was a multiple of the technical face so that frames from the two resulting videos could be matched to compare the bloodstain on the technical rear to that on the technical face. Between 90 and $100 \mathrm{~ms}$ of high-speed video was saved for each impact.

\begin{tabular}{|l|l|l|}
\hline & V12 (technical rear) & V7 (technical face) \\
\hline Resolution rate (frames per & $512 * 512$ & $512 * 512$ \\
\hline $\begin{array}{l}\text { Frame recond) } \\
\text { seconosure }(\mu \mathrm{s})\end{array}$ & 60 & 5000 \\
\hline Exposule & 80 \\
\hline
\end{tabular}

Table 2 the setting for the high speed cameras.

In order to simulate body temperature, the horse blood was heated in a water bath to $37^{\circ} \mathrm{C}$. The calico was placed on the clear polycarbonate surface above the phantom V12 camera before bloodstains were created by dropping the blood vertically using a Pasteur pipette. Three different heights were

${ }^{4}$ E-H Cavendish Courtyard, Sallow Road, Weldon North Industrial Estate, Corby, Northants. www.sglab.co.uk 
used; $200 \mathrm{~mm}, 1000 \mathrm{~mm}$ and $2000 \mathrm{~mm}$. The resulting high-speed video was analysed using Phantom Camera Control software ${ }^{5}$ to measure the impact velocity and drop diameter. The mean drop diameter was $3.8 \pm 0.17 \mathrm{~mm}$, cross-sectional area was $11.3 \mathrm{~mm}^{2}$. The velocities were $1.9 \pm 0.06 \mathrm{~ms}^{-1}, 4.2 \pm 0.06$ $\mathrm{ms}^{-1}$ and $5.7 \pm 0.07 \mathrm{~ms}^{-1}$. Five repeats were done at each height on each fabric, resulting in a total of 45 specimens. The areas of the technical rear bloodstains $1 \mathrm{~ms}$ after impact were obtained by drawing around the visible bloodstain using the freehand drawing tool in Image $J^{6}$ (figure 2). The dry technical face and technical rear bloodstains were measured using the inbuilt tools in ImageJ.

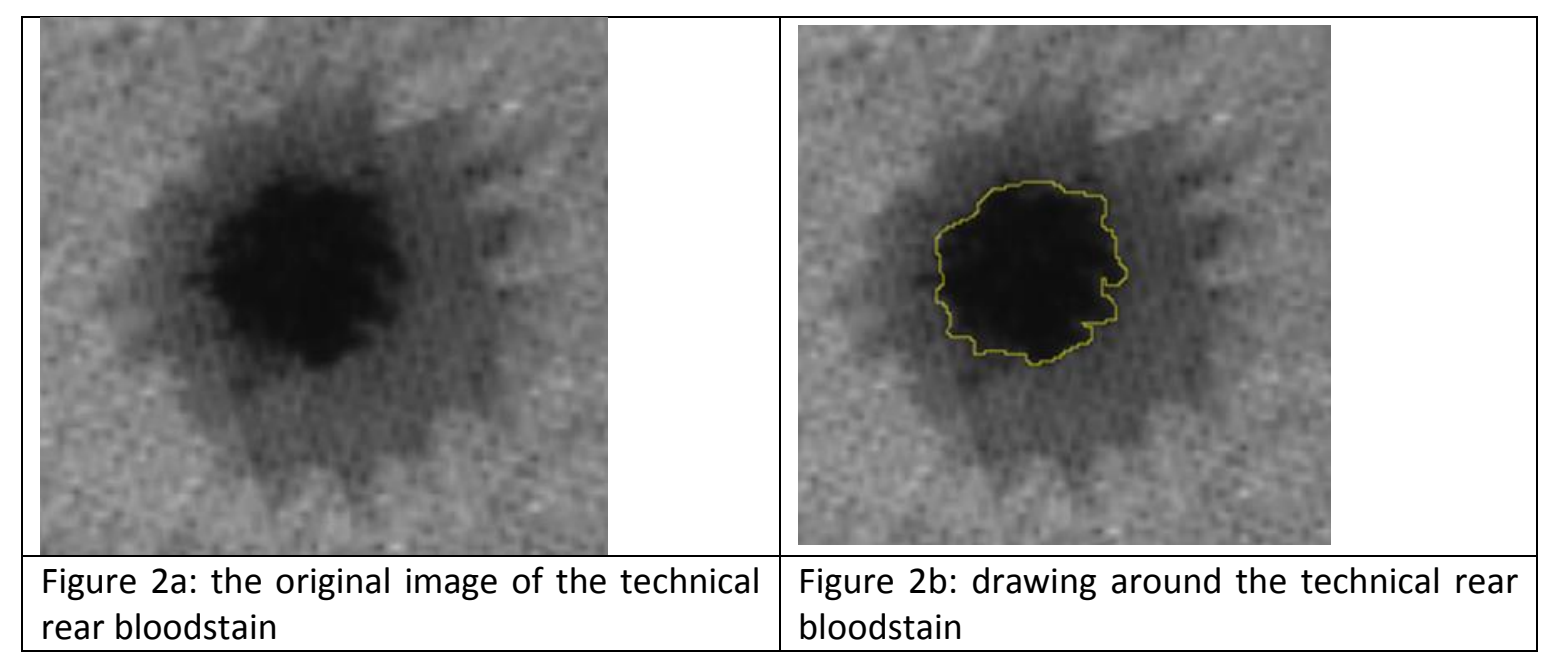

Figure $\mathbf{2}$ measuring the area of an example technical rear bloodstain image. The darkest central area is where the blood has passed through to the technical rear of the fabric, while the light surrounding area is blood on the surface of the fabric.

\section{Results}

\section{Impact dynamics}

Example high-speed video stills of the technical face and technical rear from a drop impacting the light calico at $4.2 \mathrm{~ms}^{-1}$ are given in figure 3. The marked area was where the blood was visible on the technical rear of the fabric, and is the area measured in figure $2 \mathrm{~b}$. Surrounding this, the blood which was spreading on the technical face of the fabric can be seen.

\footnotetext{
${ }^{5}$ https://www.phantomhighspeed.com/resourcesandsupport/phantomresources/pccsoftware page accessed $27^{\text {th }}$ September 2018.

${ }^{6}$ ImageJ is a public domain Java image processing programme https://imagej.nih.gov/ij/ page accessed $27^{\text {th }}$ September 2018.
} 


\begin{tabular}{|c|c|c|c|}
\hline $\begin{array}{l}\text { Time } \\
\text { following } \\
\text { when } \\
\text { impact can } \\
\text { be seen on } \\
\text { the } \\
\text { technical } \\
\text { face }\end{array}$ & Technical face & $\begin{array}{l}\text { Technical rear with blood } \\
\text { which has passed through } \\
\text { the fabric indicated }\end{array}$ & $\begin{array}{l}\text { Technical } \\
\text { rear } \\
\text { bloodstain } \\
\text { area }\end{array}$ \\
\hline $\begin{array}{l}\text { Impact } \\
\text { visible on } \\
\text { technical } \\
\text { face }\end{array}$ & & & $7 \mathrm{~mm}^{2}$ \\
\hline $0.2 \mathrm{~ms}$ & & & $16 \mathrm{~mm}^{2}$ \\
\hline $0.335 \mathrm{~ms}$ & & & $21 \mathrm{~mm}^{2}$ \\
\hline $0.6 \mathrm{~ms}$ & & & $21 \mathrm{~mm}^{2}$ \\
\hline
\end{tabular}




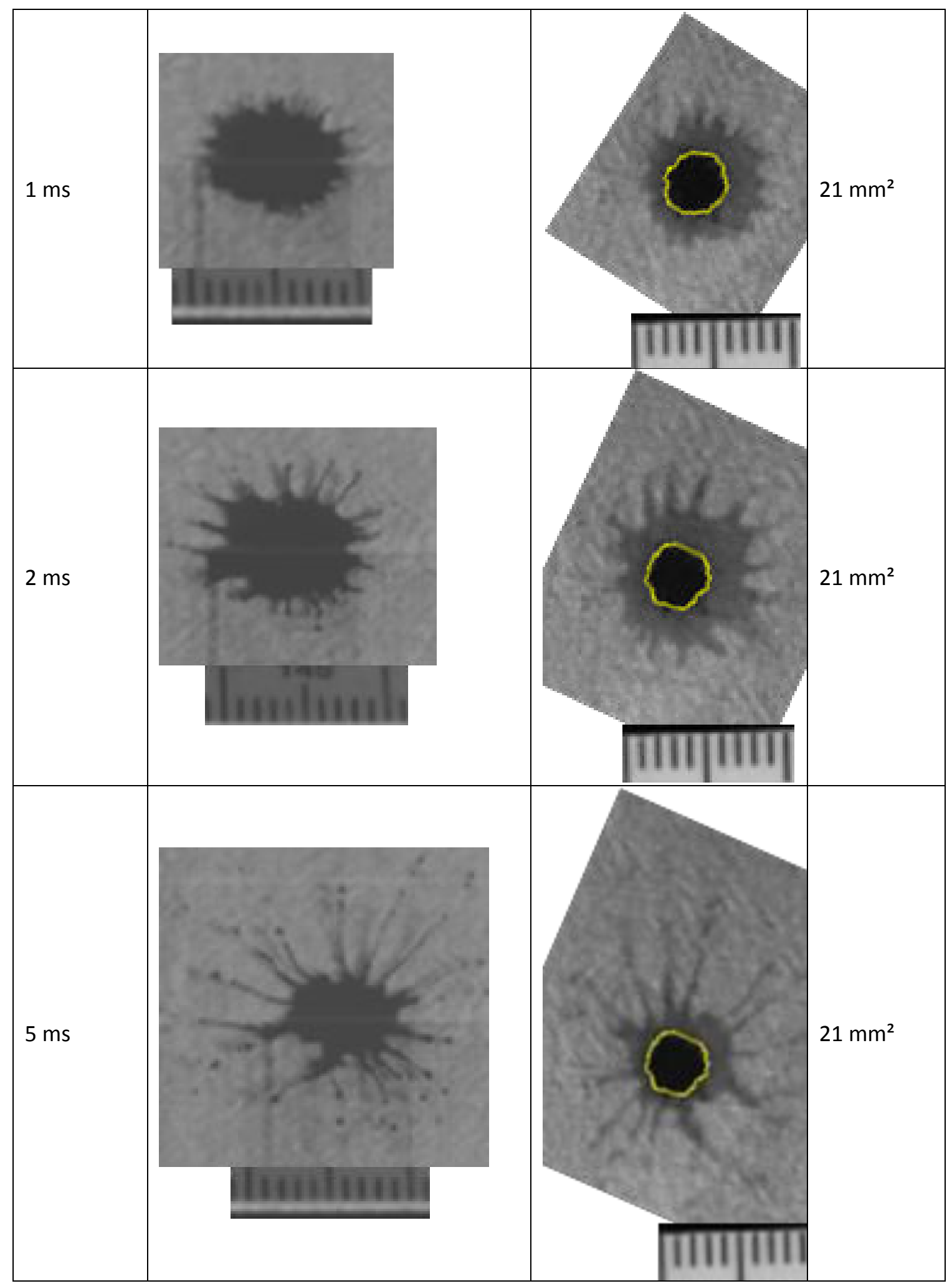




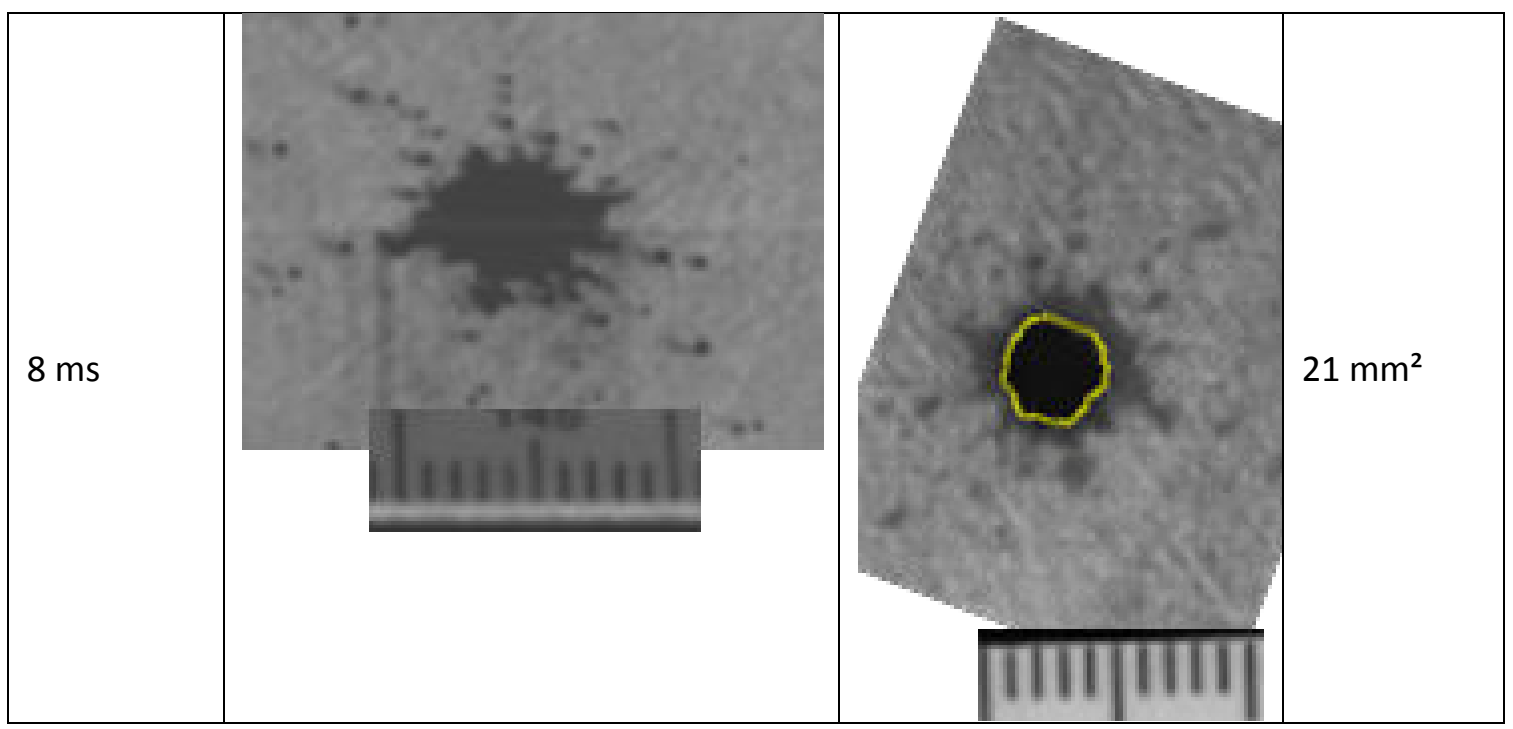

Figure 3 impact stages on the technical face and technical rear of the light calico of a single specimen from $4.2 \mathrm{~ms}^{-1}$. The technical face and technical rear bloodstains are orientated to the same angle but appear as mirror images owing to being filmed from the top and bottom respectively. The additional technical rear image at $0.335 \mathrm{~ms}$ is between two technical face frames. Scale is $\mathbf{2 0} \mathbf{m m}$.

On the technical face of the fabric by $0.2 \mathrm{~ms}$ the blood drop was spreading laterally in all directions across the surface of the fabric (figure 3). By 1 ms following impact (figure 3) ligaments began to form, which continued to grow until $5 \mathrm{~ms}$. At this point the main bloodstain began to retract, with satellite stains breaking off from the end of the ligaments. By $8 \mathrm{~ms}$ (figure 3) the blood drop settled, with spines and satellite stains formed around the edge of the bloodstain. For the following $67 \mathrm{~ms}$ of high speed video there was no further change in the technical face bloodstain. From this point, previous work [4] has indicated the blood may wick along the yarns, further increasing the bloodstain area.

On the technical rear of the fabric, the frame speed for the high speed camera was faster than for the technical face, every $0.067 \mathrm{~ms}$ compared to every $0.2 \mathrm{~ms}$ for the technical face camera. On the example specimen shown in figure 3, blood appeared on the technical rear of the fabric in the highspeed video on the frame prior to the frame when impact could be seen in the technical face video. If impact occurred at the earliest point between the two frames on the technical face camera, the time for the blood to reach the technical rear of the fabric may have taken up to $0.134 \mathrm{~ms}$. This was not consistent across all specimens. On the light calico, the longest potential time from impact to blood appearing on the technical rear was between $0.5 \mathrm{~ms}$ and $0.7 \mathrm{~ms}$.

The amount of blood on the technical rear of the fabric increased from $8 \mathrm{~mm}^{2}$ to $21 \mathrm{~mm}^{2}$ by $0.335 \mathrm{~ms}$ after impact (figure 3). This occurred as the blood on the technical face of the fabric spread laterally. The longest time taken for a technical rear bloodstain to reach maximum area was $0.8 \mathrm{~ms}$, which was a medium calico specimen. Following this, although the blood on the surface of the fabric was still spreading and retracting, there was no further increase in the size of the bloodstain on the technical rear in the remaining $45 \mathrm{~ms}$ of high speed video.

\section{Effect of fabric mass per unit area}

An example technical rear bloodstain for each fabric at each velocity is shown in figure 4 . This shows that the amount of blood which was visible on the technical rear of the fabric $1 \mathrm{~ms}$ after impact varied depending on the mass per unit area of the fabric and the impact velocity of the blood drop. 


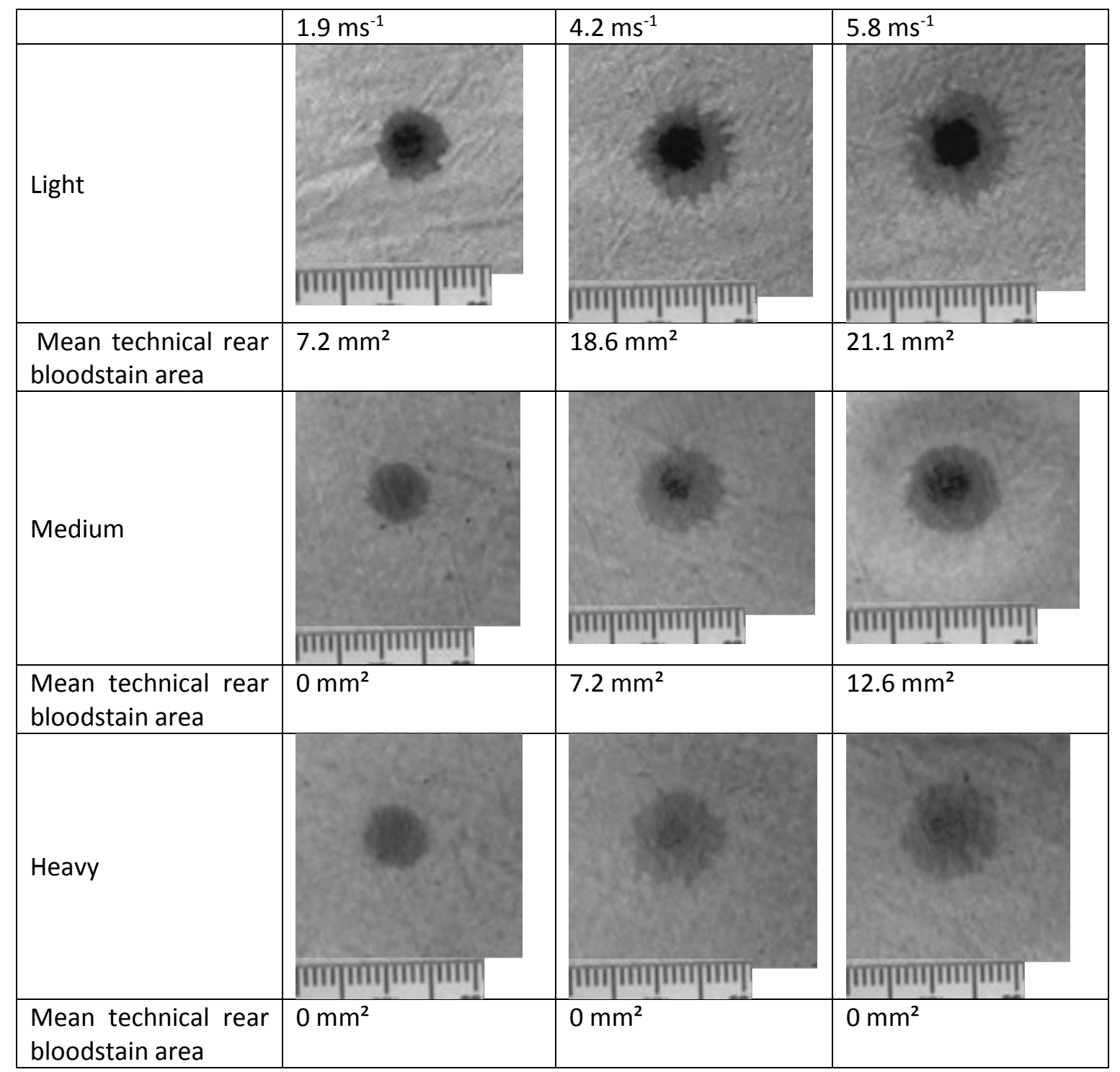

Figure 4 a typical example of a high speed video still from each fabric and each velocity from 1 ms after impact. The light areas surrounding the darker technical rear bloodstains is the blood on the surface of the fabric, which can be seen from the technical rear. Scale is $\mathbf{2} \mathbf{c m}$.

For the light calico, blood was visible on the technical rear of the fabric at all three velocities (figure 4) on the high speed video. For the slowest velocity $\left(1.9 \mathrm{~ms}^{-1}\right)$ blood was visible on the technical rear of the fabric, for only four out of the five specimens, by between 0.2 to $0.67 \mathrm{~ms}$ following impact. For the fastest velocity $\left(5.8 \mathrm{~ms}^{-1}\right)$, blood was visible on the technical rear between $0.067 \mathrm{~ms}$ and $0.268 \mathrm{~ms}$. The mean technical rear bloodstain was statistically significantly smaller $\left(F_{2,12}=22.89, p \leq 0.01\right)$ for the specimens from $1.9 \mathrm{~ms}^{-1}\left(7.2 \mathrm{~mm}^{2}\right)$ than for the other two velocities $\left(4.2 \mathrm{~ms}^{-1}: 18.6 \mathrm{~mm}^{2} ; 5.8 \mathrm{~ms}^{1}\right.$ : $21.1 \mathrm{~mm}^{2}$ ).

For the medium calico the amount of blood which was visible on the technical rear of the fabric by 1 ms after impact increased with the increase in impact velocity (figure 4). The mean technical rear bloodstain area from $4.2 \mathrm{~ms}^{-1}$ was statistically significantly smaller than that from $5.8 \mathrm{~ms}^{-1}\left(t_{8}=-3.211\right.$, $p \leq 0.05$ ). From $4.2 \mathrm{~ms}^{-1}$, blood was visible on the technical rear by between 0.268 to $0.469 \mathrm{~ms}$ following impact. For the fastest velocity $\left(5.8 \mathrm{~ms}^{-1}\right)$ blood was visible by between 0.067 and $0.335 \mathrm{~ms}$, with just one specimen taking $0.67 \mathrm{~ms}$. The technical rear bloodstains were patchier for the medium 
calico than the light calico (figure 4), as blood did not reach the technical rear of the medium calico across the entire bloodstain.

For the heavy calico, no blood was visible on the technical rear of the fabric within the high speed video for any of the three impact velocities (figure 4). Although there is a small area of slightly darker blood visible in the centre of the heavy calico video stills, this appears to be where blood has penetrated into the fabric, but not all the way through to the technical rear.

Dry technical face and technical rear area

The mean dry technical face and technical rear bloodstain areas for all three fabrics and velocities are given in table 3 , with the areas from all specimens plotted in figure 5 . Both the mean dry technical face and dry technical rear bloodstain areas for all three fabrics increased with velocity. At all velocities, the light calico had the largest mean dry technical face and technical rear bloodstains, and the medium had the smallest. An example dry technical rear bloodstain for each fabric from $4.2 \mathrm{~ms}^{-1}$ is given in figure 6 .

\begin{tabular}{|c|c|c|c|c|c|c|}
\hline \multirow{3}{*}{$\begin{array}{l}\text { Velocity } \\
\left(\mathrm{ms}^{-1}\right)\end{array}$} & \multicolumn{6}{|c|}{ Dry bloodstain areas $\left(\mathrm{mm}^{2}\right)$} \\
\hline & \multicolumn{2}{|l|}{ Light calico } & \multicolumn{2}{|c|}{ Medium calico } & \multicolumn{2}{|c|}{ Heavy calico } \\
\hline & $\begin{array}{l}\text { Technical } \\
\text { face }\end{array}$ & $\begin{array}{l}\text { Technical } \\
\text { rear }\end{array}$ & $\begin{array}{l}\text { Technical } \\
\text { face }\end{array}$ & $\begin{array}{l}\text { Technical } \\
\text { rear }\end{array}$ & $\begin{array}{l}\text { Technical } \\
\text { face }\end{array}$ & $\begin{array}{l}\text { Technical } \\
\text { rear }\end{array}$ \\
\hline 1.9 & $39.4 \pm 8.2$ & $37.3 \pm 8.7$ & $20.5 \pm 2.9$ & $6.7 \pm 4.4$ & $29.7 \pm 7.1$ & $15 \pm 9.2$ \\
\hline 4.2 & $57.9 \pm 8.6$ & $52 \pm 9.9$ & $36.7 \pm 3.5$ & $14.6 \pm 8.1$ & $51.3 \pm 8.4$ & $23.3 \pm 7.7$ \\
\hline 5.8 & $70.4 \pm 12.6$ & $58.3 \pm 8.5$ & $49.3 \pm 3.4$ & $28.9 \pm 2.7$ & $58.9 \pm 8.2$ & $37.2 \pm 5.3$ \\
\hline
\end{tabular}

Table 3 the mean and standard deviation dry technical face and technical rear bloodstains for all fabrics and velocities. 


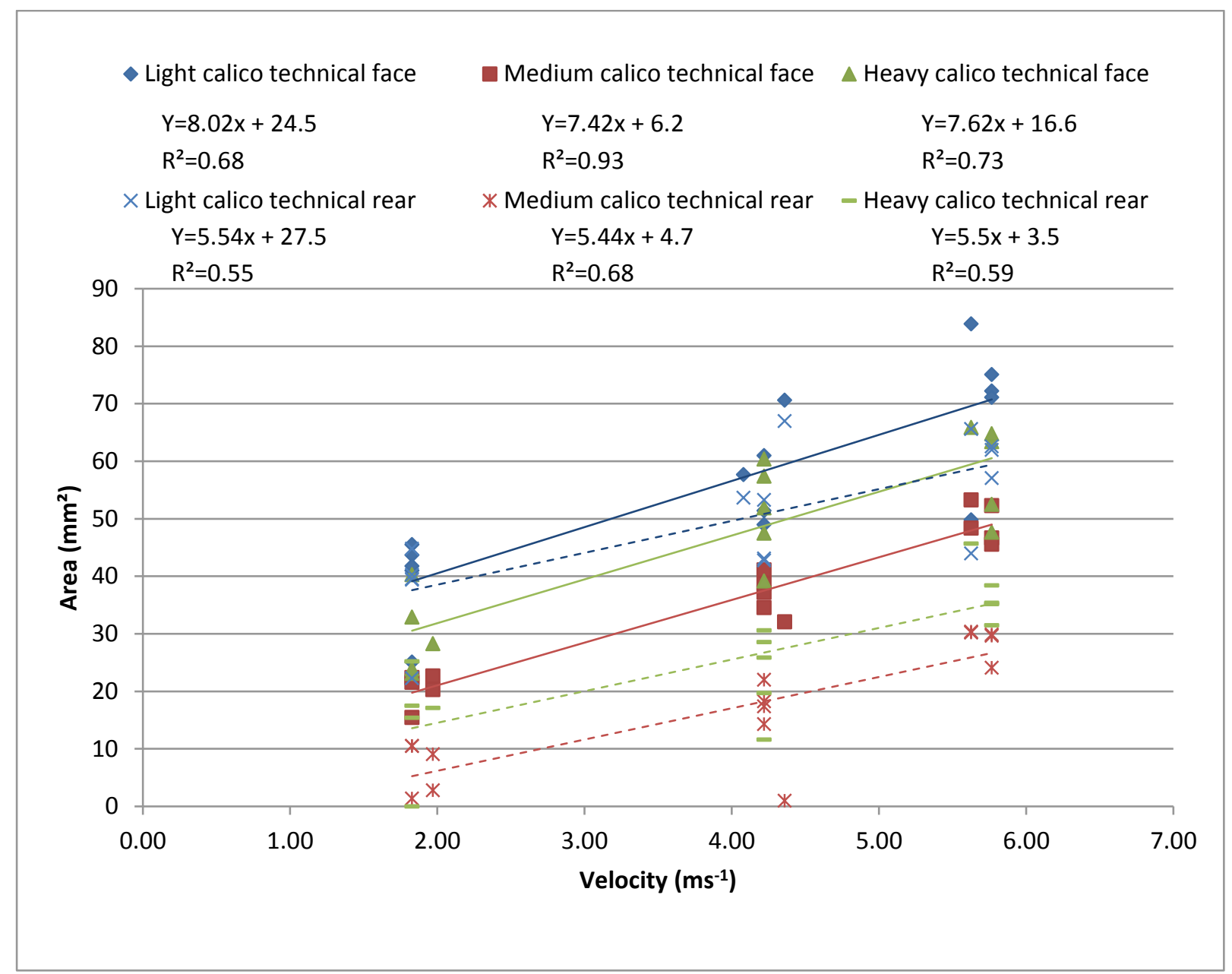

Figure 5 the dry technical face and technical rear areas for all specimens.

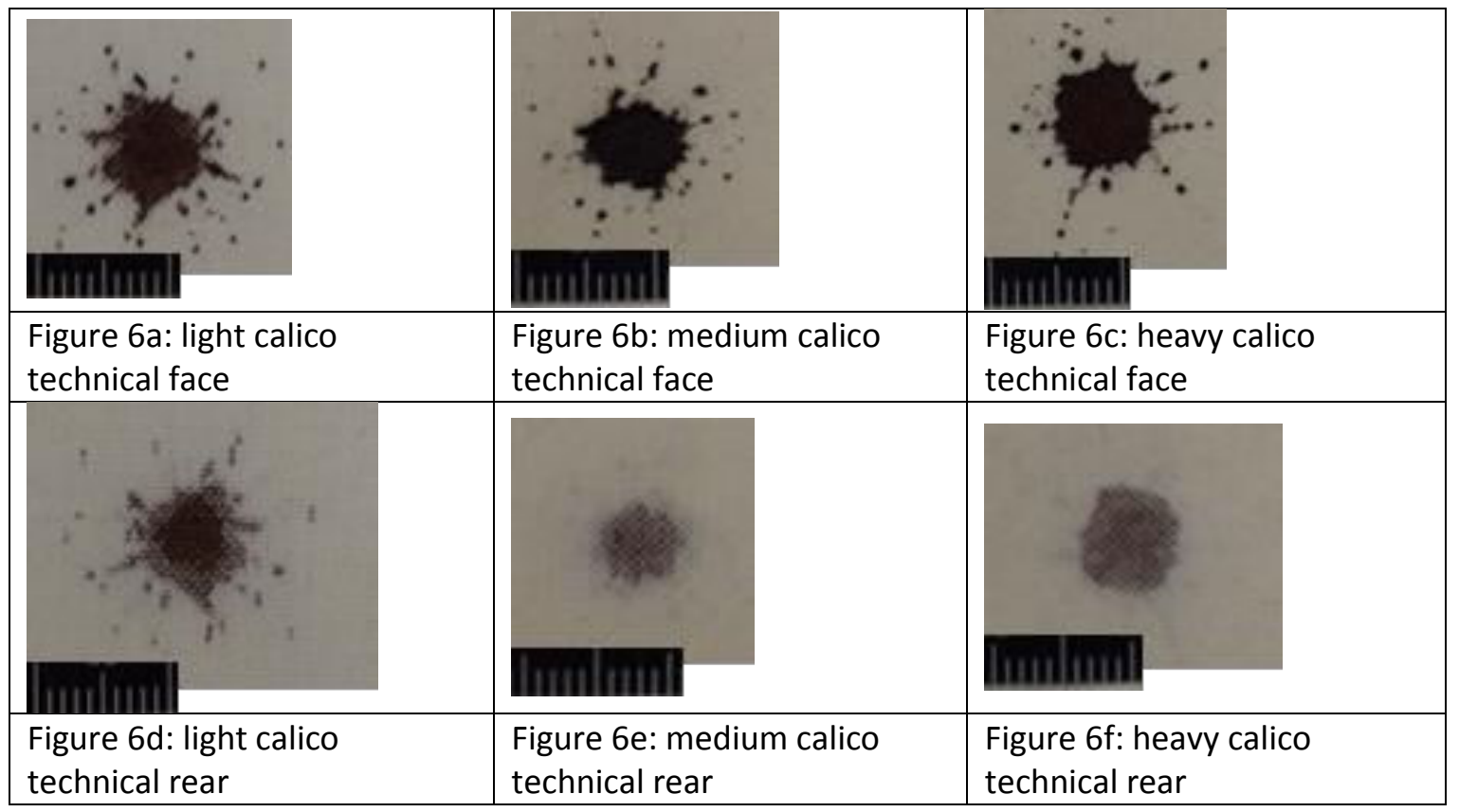

Figure 6 an example dry technical face and technical rear bloodstain on each fabric from $4.2 \mathrm{~ms}^{-1}$. Scale is $1 \mathrm{~cm}$. 


\section{Discussion}

The technical face and technical rear of three mass per unit areas of fabric were filmed using high speed video during blood drop impacts at three impact velocities. This was undertaken in an attempt to improve understanding of what was happening on the technical rear of the fabric, while the blood drop was spreading and receding on the technical face.

The impact dynamics on the technical face of the fabric in the current work agreed with those seen in previous work [4]. Briefly, the blood drop spread on the surface of the fabric, a crown formed and broke up into ligaments and satellites, before the drop retracted. However, the only mention in Williams et al. [4] of what may have occurred on the technical rear of the fabric was when a blood drop was allowed to fall $10 \mathrm{~mm}$ onto $100 \%$ cotton plain woven and single jersey fabrics. Very little wicking was seen to occur in the first 500 ms following impact [4]. The suggested reason for this was the time taken for penetration of the blood into the fabric prior to any in-plane wicking occurring. However, this was only mentioned when the blood drop impacted with very little velocity, and not in terms of the greater velocities also investigated in this previous work.

Penetration is used to describe the movement of blood vertically from the technical face to the technical rear of the fabric. On the light calico, blood had penetrated through to the technical rear of the fabric by as little $0.067 \mathrm{~ms}$ after impact. During the first $0.335 \mathrm{~ms}$ the technical rear bloodstain increased in area as the blood spread laterally on the technical face (figure 3). The penetration of the blood caused the initial increase in bloodstain area on the technical rear, which always remained smaller than the technical face bloodstain (figure 3 ) on this particular fabric. From an impact velocity of $1.9 \mathrm{~ms}^{-1}$, the mean technical rear bloodstain area on the light calico $1 \mathrm{~ms}$ after impact (figure 4, 7.2 $\mathrm{mm}^{2}$ ) was smaller than that of the cross-sectional area of the impact drop $\left(11.3 \mathrm{~mm}^{2}\right)$. Therefore, the blood was only able to penetrate where the volume of blood was at its greatest. As the velocity increased, although the mean technical rear bloodstain area increased beyond that of the crosssectional area of the blood drop, the area of the technical rear bloodstain only increased for the first $0.335 \mathrm{~ms}$ following impact (figure 3 ). As the blood spread laterally across the surface of the fabric there was no longer enough volume for the blood to penetrate to the technical rear of the fabric, therefore no further increase in technical rear bloodstain area was seen at this point.

Whilst the technical rear bloodstain had ceased to spread by no later than $0.8 \mathrm{~ms}$ following impact, the blood on the technical face of the bloodstain continued to spread, before retracting and producing satellite stains and spines. The drop impact dynamics on the technical face of the fabric did not conclude until $8 \mathrm{~ms}$. For the following $67 \mathrm{~ms}$ of high speed video there was no further change in the bloodstain. Following this lateral wicking along the yarns occurred.

It has previously been suggested $[3,4]$ that blood on the technical rear of a fabric on a hard surface spreads independently of the blood on the technical face. The movement of blood on the technical rear of the fabric is caused by pooling and spreading on the hard surface, before wicking back into the fabric. This phenomenon has been mentioned as a reason for not using a hard backing in blood pattern research. During the initial penetration of the blood through to the technical rear, the period of time over which the technical rear blood was increasing (up to the first $0.8 \mathrm{~ms}$ after impact), compared to the length of time over which the impact dynamics on the technical face were occurring $(8 \mathrm{~ms})$ suggests that the effect on the technical face bloodstain would be minimal. Following this, wicking did increase the technical rear bloodstain area. Looking at figure $6 \mathrm{~d}$, the area in the centre of the 
bloodstain is a solid bloodstain, where the blood penetrated through to the technical rear of the fabric at impact. The area surrounding this is where the blood wicked along the yarns, primarily the warp yarns, increasing the technical rear bloodstain area. Any wicking which did occur on the technical rear was therefore movement along the yarns, rather than movement back from the hard surface beneath.

The amount of blood which penetrated through to the technical rear of the fabric was both fabric and velocity dependent (figure 4). The penetration through to the technical rear of the fabric at impact occurred to the greatest extent on the light calico owing to the low yarn linear density (14 and 18 tex) leading to a thin fabric $(0.38 \mathrm{~mm})$ with large inter-yarn spaces. The blood therefore could easily penetrate both the inter-yarn spaces and the yarns. For the light calico, the mean dry technical rear areas $\left(37.3,52\right.$ and $58.3 \mathrm{~mm}^{2}$, table 3 ) were larger than those measured $1 \mathrm{~ms}$ following impact (7.2, 18.6 and $21.1 \mathrm{~mm}^{2}$, figure 4 ) as the blood wicked along the yarns.

The medium calico had a greater yarn linear density ( 33 and 31 tex) than the light calico, resulting in a thicker fabric $(0.46 \mathrm{~mm})$ with smaller inter-yarn spaces, reducing the ease with which the blood could penetrate to the technical rear of the fabric. At $1.9 \mathrm{~ms}^{-1}$, the blood drop did not penetrate to the technical rear, as there was not enough kinetic energy at impact to force the blood into the inter-yarn spaces (figure 4). From $4.2 \mathrm{~ms}^{-1}$ and $5.8 \mathrm{~ms}^{-1}$ the increase in kinetic energy at impact forced a small amount of blood to penetrate to the technical rear of the fabric (figure 4). The greater yarn linear density and higher twist (756 turns per metre) of the medium than the light calico also reduced the ease with which the blood could then wick into the yarns. Increased twist has previously been shown to reduce wicking in yarns [17]. This resulted in the patchier technical rear bloodstains for the medium compared to the light calico, as well as the smallest dry technical rear bloodstain areas (table 3 ).

The heavy calico had the greatest yarn linear density ( 43 and 47 tex) and therefore the smallest interyarn spaces of the three fabrics investigated in this work. The blood did not penetrate the entire thickness of the fabric at impact from any of the impact velocities investigated. The blood drop did not reach a sufficient kinetic energy with which to force the blood into the inter-yarn spaces and through to the technical rear of the fabric at impact. However, the blood was able to wick through, or around, the yarns before drying. This meant that when the dry bloodstain was examined, there was a bloodstain on the technical rear of the fabric (figure $6 f$, table 3 ). The dry technical rear bloodstain on the heavy calico was larger than that of the medium calico owing to the differences in yarn twist levels (650 $\times 624$ tpm and $756 \times 756$ tpm for the heavy and medium calicos respectively). The lower twist level for the heavy calico meant once the blood was able to wick into the intra-yarn spaces, they were a more optimum size for wicking than those of the medium calico. This increased the dry bloodstain areas beyond those of the medium calico.

\section{Conclusion}

Bloodstains formed on the technical face and technical rear of three $100 \%$ cotton plain woven fabrics with different yarn linear densities were filmed using high speed video. The aim was to determine if dynamics that occur on the technical rear of the fabric as the blood impacts affects what occurs on the technical face. The results showed that for the fabrics studied, the effect of the technical rear bloodstain on the dynamics on the technical face would therefore be minimal.

On the light calico $\left(85.1 \mathrm{~g} / \mathrm{m}^{2}\right)$, blood was visible on the technical rear of the fabric within as little as $0.067 \mathrm{~ms}$ of impact. As the blood was spreading on the technical face of the fabric, blood continued 
to penetrate through to the technical rear. The maximum technical rear bloodstain area from penetration was reached by no more than $0.8 \mathrm{~ms}$ after impact, as the blood was too spread out on the surface of the fabric for further penetration. The spreading and retraction of the blood drop on the technical face continued for $8 \mathrm{~ms}$. The effect of the technical rear bloodstain on the dynamics on the technical face would therefore be minimal.

Whether blood was visible on the technical rear of the fabric within the high speed video was dependent on both the fabric and the impact velocity of the drop. For the heavy calico $\left(224.6 \mathrm{~g} / \mathrm{m}^{2}\right)$, no blood penetrated through to the technical rear of the fabric at impact, for the medium calico (163.5 $\mathrm{g} / \mathrm{m}^{2}$ ) it was velocity-dependent, and for the light calico blood penetrated through to the technical rear of the fabric at impact at all velocities. Once the impact dynamics were complete $(8 \mathrm{~ms})$ and following the conclusion of the high speed video, the blood wicked through and along the yarns, resulting in a dry technical rear bloodstain on all fabrics at all velocities.

\section{Ethical Statement}

All applicable international, national and institutional guidelines for the care and use of animals were followed.

\section{References}

[1] T.C. de Castro, M.C. Taylor, J.A. Kieser, D.J. Carr, W. Duncan, Systematic investigation of drip stains on apparel fabrics: The effects of prior-laundering, fibre content and fabric structure on final stain appearance, Forensic Sci. Int. 250 (2015) 98-109.

doi:10.1016/j.forsciint.2015.03.004.

[2] T.C. De Castro, D.J. Carr, M.C. Taylor, J.A. Kieser, W. Duncan, Drip bloodstain appearance on inclined apparel fabrics: Effect of prior-laundering, fibre content and fabric structure, Forensic Sci. Int. 266 (2016) 488-501. doi:10.1016/j.forsciint.2016.07.008.

[3] X. Li, J. Li, S. Michielsen, Effect of yarn structure on wicking and its impact on bloodstain pattern analysis (BPA) on woven cotton fabrics, Forensic Sci. Int. 276 (2017) 41-50. doi:10.1016/j.forsciint.2017.04.011.

[4] E.M.P. Williams, M. Dodds, M.C. Taylor, J. Li, S. Michielsen, Impact dynamics of porcine drip bloodstains on fabrics, Forensic Sci. Int. 262 (2016) 66-72.

doi:10.1016/j.forsciint.2016.02.037.

[5] J.Y.M. Chang, S. Michielsen, Effect of fabric mounting method and backing material on bloodstain patterns of drip stains on textiles, Int. J. Legal Med. (2016). doi:10.1007/s00414015-1314-z.

[6] L. Dicken, C. Knock, S. Beckett, T.C. de Castro, T. Nickson, D.J. Carr, The use of micro computed tomography to ascertain the morphology of bloodstains on fabric, Forensic Sci. Int. 257 (2015) 369-375. doi:10.1016/j.forsciint.2015.10.006.

[7] J. Li, X. Li, S. Michielsen, Alternative method for determining the original drop volume of bloodstains on knit fabrics, Forensic Sci. Int. 263 (2016) 194-203.

doi:10.1016/j.forsciint.2016.04.018. 
[8] T. De Castro, T. Nickson, D. Carr, C. Knock, Interpreting the formation of bloodstains on selected apparel fabrics, Int. J. Legal Med. 127 (2013) 251-258. doi:10.1007/s00414-0120717-3.

[9] British Standards Institution, Textiles - Domestic washing and drying procedures for textile testing, BS EN ISO 6330. (2012).

[10] British Standards Institution, Textiles. Sampling of fibres, yarns and fabrics for testing, BS EN 12751. (1999).

[11] British Standards Institution, Textiles - Standard atmospheres for conditioning and testing, BS EN ISO 139. (2005).

[12] British Standards Institution, Textiles - Determination of thickness of textiles and textile products, BS EN ISO 5084. (1997).

[13] British Standards Institution, Textiles - Woven fabrics - Determination of mass per unit length and mass per unit area, BS 2471. (2005).

[14] British Standards Institution, Textiles - Woven fabrics - Construction - Methods of analysis Part 2: Determination of the number of threads per unit length, BS EN 1049-2. (1994).

[15] D.V. Christman, A Study to Compare Animal Blood to Human Blood Product, Int. Assoc. Bloodstain Pattern Anal. Assoc. Crime Scene Reconstr. Jt. Train. Conf. (1997) 16.

[16] M.A. Raymond, E.R. Smith, J. Liesegang, The physical properties of blood--forensic considerations., Sci. Justice. 36 (1996) 153-160. doi:10.1016/S1355-0306(96)72590-X.

[17] A.B. Nyoni, D. Brook, Wicking mechanisms in yarns - the key to fabric wicking performance, J. Text. Inst. 97 (2006) 119-128. doi:10.1533/joti.2005.0128. 